\title{
Abstract
}

\section{New Homeopathic Preparations in Practice}

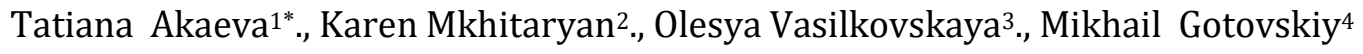 \\ 1,2,4 - Limited Liability Company Center of Intellectual Medical Systems "IMEDIS" Moscow, Russia. \\ 3 - Medical center «Vitamed plus» Gabrovo, Bulgaria. \\ *akaeva_tatyana@mail.ru - https://orcid.org/0000-0002-9659-8080
}

Background One of the most important tasks in anti-age therapy and rejuvenation practice is the regeneration of various tissues, organs and body systems [1,2]. In this study, we evaluated the effectiveness of therapy with the new class of homeopathic and informational preparations "Triton Regeneration" and "Triton Metamorphosis" [3]. The preparations were used both in homeopathic dilutions, and in the form of control signals of the Triton (CSTrit). Aims To apply in practice the homeopathic preparations "Triton regeneration" and "Triton metamorphosis" in the form of homeopathic dilutions and control signals, in order to regenerate the organs of the tissues and systems of the patient and evaluate the results obtained. Methodology A clinical study was conducted on a group of 33-st people aged between 7-75 years of both sexes who received injuries to the skin, connective tissue and / or musculoskeletal system. The study was conducted by a multicenter method in the cities: Moscow (Russia), Gabrovo (Bulgaria), Arad (Romania). All patients gave informed consent for treatment. To write a CS and their applications, for diagnostics and therapy, and the fabrication for the necessary preparations and marker $\mathrm{CMH}$, the integrated system for electropuncture diagnostic «IMEDIS-EXPERT» was used. For the diagnosis method was used vegetative resonance test (VRT). Cultures of the biological material of the triton were used to obtain control signals triton (CSTrit) therapy. The method of bioresonance therapy (BRT) was used to adapt and administer the condition of the patient. Adaptation of the preparations to the patient's body was carried out by electron potentiation of the initial CSTrit until the VRT condition was met:

\section{CMH $\downarrow+$ Pot $^{\alpha}$ (CSTrit) $\uparrow$}

The marker $\mathrm{CMH}$-individual constitutional marker represents the sum of signals from the nodal and terminal BAT (biologically active points) located on the main chirohlyphic palm lines of the patient. Patients used the homeopathic remedies of triton and / or adapted CSTrit in individual dosage during the individual course of therapy. At subsequent receptions, a second VRT was re-examined and the degree of recovery of defects in the skin, connective tissue and / or the musculoskeletal system was assessed. Results This clinical study showed a high efficacy of homeopathic and informational preparations of CSTrit in patients who received injuries of the skin, connective tissue and / or musculoskeletal system. A pronounced positive effect was obtained in all cases of treatment of CSTrit: objective processes of regeneration of the connective tissue system of the patients were noted, in particular, restoration of its skin. In the case of treatment therapy for injuries of the musculoskeletal system, marked regeneration processes in the bone and tendon tissues were observed, confirmed by R-studies. Conclusion 1. Homeopathic remedies "Triton regeneration" and "Triton metamorphosis", as well as the control signals of the Triton (CSTrit), made using the apparatus "IMEDIS-EXPERT", are effective signals of the regeneration of body tissues, as in the case of treatment of skin and connective tissue damage, and in case of damage to bone tissue and tendons. 2. Therapy of patients with CSTrit, potentiated by the $\mathrm{CMH}$ marker, is comparable in effectiveness and end results with mono- and complex homeopathic therapy and in some cases surpasses it. 3. A new class of the preparations gives a positive result in $96 \%$ of cases. Similar results are unattainable with ordinary homeopathy.

Keywords: regeneration, triton, homeopathic preparation, informational preparation, individual complex marker of chrono-semantics (CMH)

OPEN ACCESS 
(C) International Journal of High Dilution Research.

Not for commercial purposes.

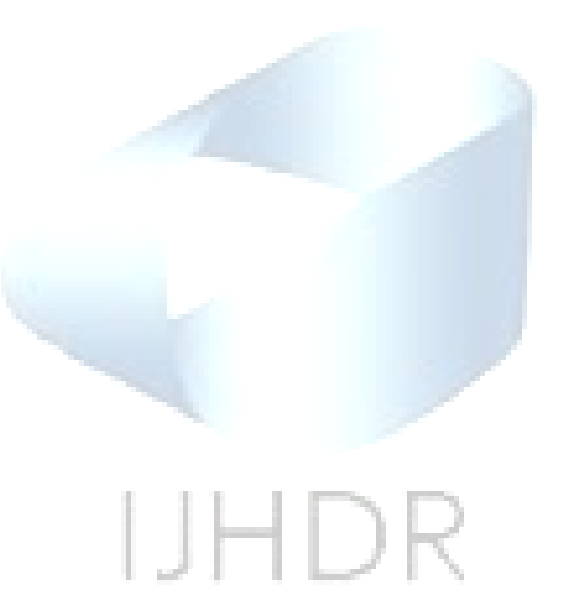

